\title{
MORPHOLOGICAL OPERATORS FOR SEGMENTATION OF HIGH CONTRAST TEXTURED REGIONS IN REMOTELY SENSED IMAGERY
}

\author{
Igor Zingman, Dietmar Saupe \\ Department of Computer and Information Science \\ University of Konstanz \\ Germany
}

\section{Karsten Lambers}

\author{
Zukunftskolleg, University of Konstanz \\ \& Institute of Archaeology, \\ Heritage Studies and Art History \\ University of Bamberg \\ Germany
}

\begin{abstract}
We develop a transformation based on morphological filters that measures the contrast of image texture. This transformation is proportional to texture contrast, but insensitive to its specific type. Though the transformation provides a high response in textured areas, it suppresses individual high contrast features that stand apart from textured areas. It can serve as an effective texture descriptor for unsupervised or supervised segmentation of textured regions, provides high accuracy of localization and does not involve heavy computations. The method is robust to variations of illumination and works on different types of images without needing to be tuned. The only parameter is a scale related parameter. We illustrate the use of the proposed method on satellite and aerial images.
\end{abstract}

Index Terms - Texture detection, texture segmentation, texture contrast descriptor, morphological filters

\section{INTRODUCTION}

Image segmentation frequently is one of the first tasks to be accomplished for discriminating between different regions in high resolution remotely sensed imagery. Texture descriptors that characterize image regions irrespectively of illumination and scene color variability are extremely important for achieving robust segmentation results. Although there is no strict definition of texture, it is usually associated with repeating image patterns or, more generally, with repeating changes of gray level. Autocorrelation, co-occurrence matrices, filter banks, wavelets, mathematical morphology, and other methods have been developed for texture characterization and employed for segmentation tasks. Some methods were designed to be invariant to texture contrast (texture strength). Contrast invariance is important for the separation of different types of textures irrespectively of contrast variations within the same texture type. On the other hand such methods can erroneously associate regions without texture, as perceived by humans, to one of the texture types with high contrast. A powerful ap- proach that attracted attention in the recent literature was developed by Ojala et al. [1], who proposed a pair of independent texture descriptors. One descriptor is an original local binary pattern descriptor related to inherent texture properties, the other descriptor relates to texture contrast. This texture contrast descriptor is based on measuring the variance of gray levels within properly defined neighborhoods. A variance based descriptor by itself is not good enough since it can produce high response close to individual features that do not form texture. Moreover, it is highly dependent on the size of the analysis window and blurs the borders of textured regions (see Fig. 2(b) for an example).

In this paper we introduce an illumination invariant texture contrast descriptor that does not suffer from the above disadvantages. This descriptor is intended to separate high contrast textures, irrespectively of texture type from smooth regions and individual features. It is developed on the basis of mathematical morphology, which provides a theoretically consistent nonlinear analysis that has proven to be very effective in various practical applications, including processing of remotely sensed imagery [2]. We describe attractive properties of the proposed morphological texture contrast descriptor (MTC) and show its effectiveness using high resolution remotely sensed images. We also compare the MTC to a commonly used variance based texture contrast descriptor and an alternative contrast descriptor based on differential morphological profiles (DMP)[3].

This work was motivated by our recently initiated project on detection of archeological objects in satellite images [4]. Since the objects of our interest (such as remainings of huts and cattle enclosures) lie in smooth regions, filtering out high contrast textured regions, such as urban areas, forests, and rocky areas is a necessary first step.

\section{METHODOLOGY}

We are interested in a morphological transformation that results in a high response in textured areas only, proportional 
to texture contrast and insensitive to inherent characteristics of a texture. We first refer to the texture contrast as a local contrast measured by the difference of gray levels (difference contrast). Let us denote by $f(x)$ a non-negative function which is bounded by the maximum value $M$ of the data type used and defined over the domain $\mathbf{S}$. This function may represent a non-negative 1D signal, when $\mathbf{S} \subset \mathbb{R}$, or 2D image, when $\mathbf{S} \subset \mathbb{R}^{2}$. To achieve our goals, we consider morphological transformations that are (a) invariant to a constant gray level bias, $\psi(f)=\psi(f+a)$, where $a \in \mathbb{R}$ is a constant, (b) self-complementary, as defined in [5, 6], $\psi(f)=\psi\left(f^{c}\right)=$ $\psi(M-f)$, and (c) linearly proportional to the texture contrast. The last property implies that $\psi(a f)=a \psi(f)$. A bias invariant and self-complementary transformation also satisfies the equality $\psi(f)=\psi(a-f)$, where $a \in \mathbb{R}$ is any constant.

A variance based texture contrast descriptor may readily satisfy the above three properties. An example of such a descriptor is given by

$$
[v(f)](x)=\sqrt{\frac{1}{|W|} \sum_{p \in W_{x}}(f(p)-\mu(x))^{2}},
$$

where $|W|$ denotes the area of a sliding window $W, W_{x}$ denotes the window centered at pixel $x$ and $\mu(x)$ is the average pixel value in $W_{x}$, i.e. $\mu(x)=\frac{1}{W} \sum_{q \in W_{x}} f(q)$.

As was also noted in [6], the sum of top-hat, $f-\gamma(f)$, where $\gamma$ is an opening operator, and bottom-hat, $\varphi(f)-f$, where $\varphi$ is a closing operator, provides an example of morphological self-complementary transformation. This transformation is also invariant to gray level bias. Moreover, a multi-scale extension,

$$
\psi_{1}=\max _{r=1, \ldots, n}\left(\varphi_{r}-\varphi_{r-1}\right)+\max _{r=1, \ldots, n}\left(\gamma_{r-1}-\gamma_{r}\right),
$$

where $r$ denotes a size parameter, also satisfies the above three properties. This transformation is closely related to the DMP in [3] and the maximum differences in [7]. Unlike variance based texture contrast, such a multi-scale transformation in Eq. (2) does not blur the borders of textured regions. It, however, yields a high response nearby individual features (see Fig. 2(c)), which is not in line with our goals. In this paper we define another morphological transformation $\psi_{2}$ that is based on the difference between texture envelopes obtained by means of morphological compositions, $\left[\hat{\psi}_{2}(f)\right](x)=\left[\gamma_{r} \varphi_{r}(f)\right](x)-\left[\varphi_{r} \gamma_{r}(f)\right](x)$,

$$
\left[\psi_{2}(f)\right](x)=\max \left(\left[\hat{\psi}_{2}(f)\right](x), 0\right)
$$

$\gamma_{r} \varphi_{r}(f)$ denotes morphological closing followed by opening while $\varphi_{r} \gamma_{r}(f)$ denotes opening followed by closing. These compositions, also called alternating morphological filters, are typically used for image denoising. We call the transformation in Eq. (3) the morphological texture contrast (MTC) operator. The MTC satisfies the three above properties, and, in contrast to the DMP based operator $\psi_{1}$, it produces a zero or diminished response in the vicinity of individual features. Such a behavior is illustrated in Fig. 1, where the MTC was applied to a simple 1D artificial signal. To detect texture regions size $r$ of the structuring element should be chosen to be larger than the characteristic size of texture details and the distances between them. In the following we show that the MTC transformation also has attractive localization properties when applied to real world images.

In the $2 \mathrm{D}$ case $\gamma \varphi$ is not necessarily greater or equal to $\varphi \gamma$ (there is no ordering between these filters [8]), therefore $\hat{\psi}_{2}$ can be negative. It can be shown that the negative structures, i.e. connected components with negative $\hat{\psi}_{2}$, are smaller than the size of the structuring element used. These structures are smaller than $r$ in the sense that an erosion with structuring element of the same size $r$ completely removes them. Such small negative structures are suppressed in Eq. (3), and not considered to be a part of a texture.

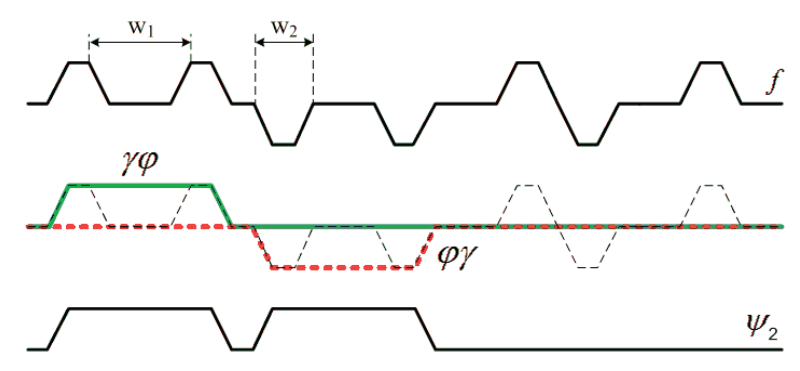

(a)

Fig. 1. Top: a signal composed of two textured regions and two individual features on the right side. $w_{1}$ is the distance between texture details, $w_{2}$ is the detail size. Middle: the green signal is a closing followed by an opening, the red dashed-line signal is an opening followed by a closing. The structuring element used is of size $w_{1}$. Bottom: The MTC transformation $\psi_{2}$. It is proportional to texture contrast (difference-contrast) and yields zero response at individual features.

Above, we have considered local contrast as measured by a difference of gray level pixel values (difference-contrast). Hereafter, we consider local contrast as measured by the gray level ratio (ratio-contrast). In accordance with the multiplicative model of image formation, image gray level values are proportional to the product of illumination and surface reflectance. The former is determined by properties of incident light while the later characterizes the observed scenery. Variation of illumination causes a proportional variation of difference-contrast. On the contrary, the ratio-contrast remains constant. Thus, a texture contrast descriptor that describes local contrast by means of gray level ratio, has the advantage of being insensitive to illumination changes. It is worth mentioning that the brightness perceived by the human visual system is also approximately logarithmically proportional to the light intensity incident on the eye. To define an 
illumination invariant texture contrast descriptor we can apply a transformation $\psi$, proportional to difference-contrast, to $\log (f)$, rather than $f$. Then $\tilde{\psi}(f)=\psi(\log (f))$ becomes (non-linearly) proportional to the ratio-contrast. Since zero values of $f$ are not allowed due to the logarithmic function, in practice they are replaced by small values.

For bias invariant and self-complementary transformation $\psi, \tilde{\psi}$ is invariant to multiplication with a constant (illumination invariant), i.e. $\tilde{\psi}(f)=\tilde{\psi}(m f), m>0$, and invariant to inversion of image gray level values, $\tilde{\psi}(f)=\tilde{\psi}(M / f)$. Such a transformation also satisfies $\tilde{\psi}(f)=\tilde{\psi}(m / f), m>0$. Particularly, the MTC transformation applied to $\log (f)$ becomes logarithmically proportional to the ratio contrast since

$$
\hat{\psi}_{2}(\log (f))=\log \frac{\gamma_{r} \varphi_{r}(f)}{\varphi_{r} \gamma_{r}(f)} .
$$

This equation is due to the property of flat ${ }^{1}$ morphological operators to commute with an increasing and continuous functions ${ }^{2}$. For example, for morphological opening we have $\gamma(\log (f))=\log (\gamma(f))$.

Fig. 3(b, e) shows an example of the illumination invariant MTC transformation $\tilde{\psi}_{2}(f)=\psi_{2}(\log (f))$ applied on the pan-chromatic satellite image Fig. 3(a) and the aerial image Fig. 3(d). The images contain high contrast textured regions, namely urban and forest areas. We can see that the MTC transformation provides a descriptor that has high values in textured regions and low values within smooth areas. The distribution of the descriptor values is highly bimodal in the examples. The segmentation of textured regions can be accomplished by simple thresholding of the descriptor values. For example, the segmentation results in Fig. 3(c, f), which are superimposed on the original image, were obtained by means of automatic Otsu thresholding [10]. It should be noted that borders of textured regions are well localized. In general situation, where the area sizes of textured and smooth regions may differ considerably, a supervised segmentation scheme can be employed. In that case, training samples of textured and smooth regions should be delineated prior to classification of image pixels. Based on the distribution of the MTC descriptor values within these training regions, an appropriate threshold can be found.

Fig. 2 shows several illumination invariant texture contrast descriptors computed for an image patch $f$ taken from the upper left part of Fig. 3(a). The variance based descriptor $\tilde{v}(f)=v(\log (f))$ in Fig. 2(b) was computed according to Eq. (1) with a sliding window of 30 pixels size. The multi-scale DMP based descriptor $\tilde{\psi}_{1}=\psi_{1}(\log (f))$ in Fig. 2(c) was computed using square structuring elements of side length $r=1, \ldots, 30$ pixels. The illumination invariant MTC descriptor $\tilde{\psi}_{2}=\psi_{2}(\log (f))$ shown in Fig. 2(d) and Fig. 3(b,

\footnotetext{
${ }^{1}$ Flat operators are operators invariant to threshold decomposition [5]. Morphological flat operators can be obtained using flat structuring elements.

${ }^{2}$ Increasing and continuous functions are frequently termed anamorphosis [9].
}

e) was computed with a square structuring element of 30 pixels size. Contrary to the variance based and the DMP based descriptors, it produces a diminished response at individual image features. It also does not extend the borders of textured regions as in the case with the variance based descriptor.

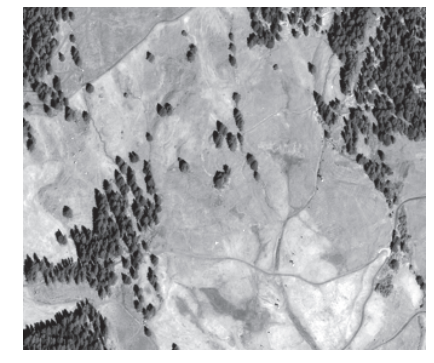

(a)

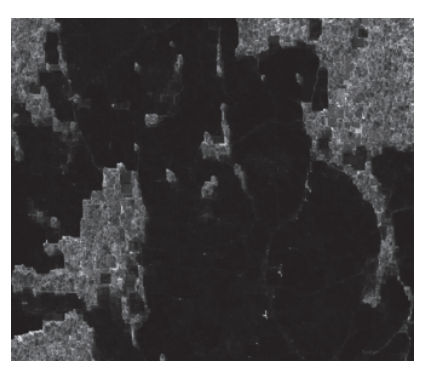

(c)

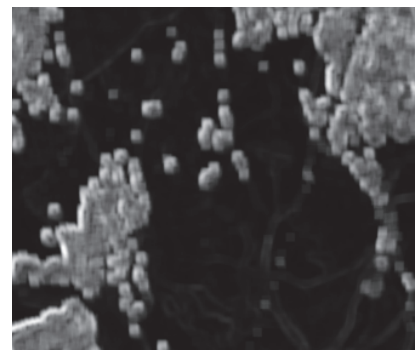

(b)

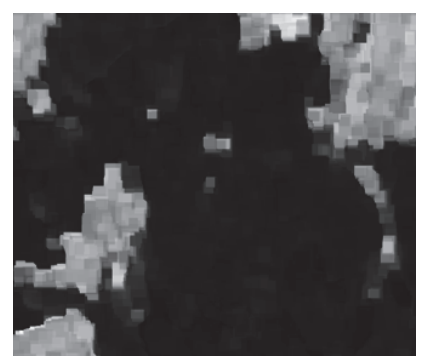

(d)
Fig. 2. (a) A patch (1360x1160 pixels) of image taken from the upper left part of Fig. 3(a). (b) Variance based illumination invariant texture contrast descriptor $\tilde{v}(f)$. (c) DMP based illumination invariant texture contrast descriptor $\tilde{\psi}_{1}(f)$. (d) Illumination invariant MTC descriptor $\tilde{\psi}_{2}(f)$

\section{CONCLUSIONS}

We presented an illumination invariant, morphological texture contrast (MTC) descriptor. The experiments showed that the MTC descriptor is effective for localization and segmentation of high contrast textured regions. Though a single-size structuring element is used, the descriptor provides attractive segmentation of different landscapes in satellite or aerial images of half a meter resolution. Incorporation of structuring elements of multiple sizes may provide superior results in cases where image textures are at extremely different scales. The descriptor is not intended to separate between different textures, since it carries only contrast information. However, it can serve as an additional descriptor combined with other descriptors which relate to inherent properties of a texture. The resulting feature vector can be used to classify textures in images, while not being confused by neither smooth regions nor individual features. 
(a)

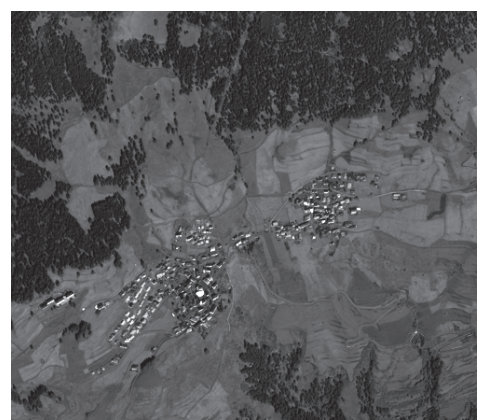

(d)

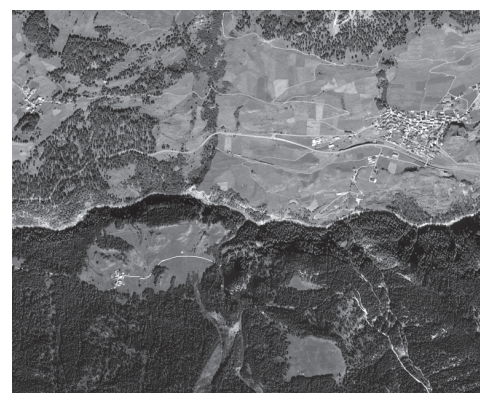

(b)

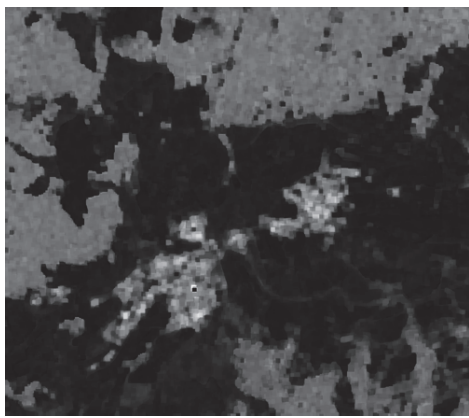

(e)

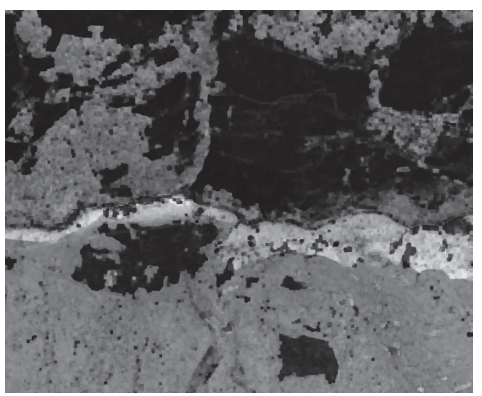

(c)

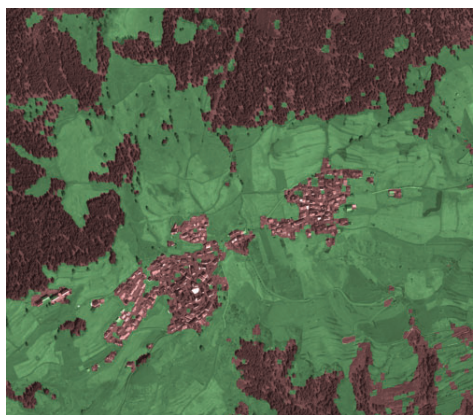

(f)

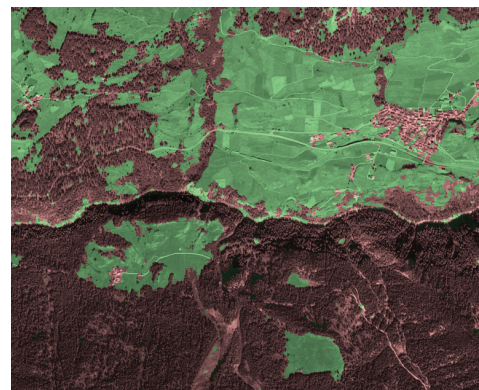

Fig. 3. a. Pan-chromatic image of $4000 \times 3500$ pixel size and $0.5 \mathrm{~m} /$ pixel resolution captured by the GeoEye- 1 satellite (C) GeoEye 2011, distributed by e-GEOS). d. Aerial SWISSTOPO image of 6100x5000 pixel size and 0.5m/pixel resolution. Scenery in both images includes high contrast textured regions (urban and forest areas), and comparably smooth field areas. b. and e. Illumination invariant MTC descriptor. c. and $\mathrm{f}$. The segmentation result superimposed on the original image was obtained by automatic thresholding of the illumination invariant MTC descriptor. Brownish areas correspond to high contrast textured regions.

\section{Acknowledgments}

This work was funded by the Zukunftskolleg, University of Konstanz and by the Interreg IV Program "Alpenrhein - Bodensee - Hochrhein". It was also partially supported by the DFG Research Training Group GK-1042 "Explorative Analysis and Visualization of Large Information Spaces", University of Konstanz.

\section{REFERENCES}

[1] T. Ojala, M. Pietikäinen, and T. Mäenpää, "Multiresolution gray-scale and rotation invariant texture classification with local binary patterns," IEEE Trans. Pattern Anal. Mach. Intell., vol. 24, pp. 971-987, July 2002.

[2] P. Soille and M. Pesaresi, "Advances in mathematical morphology applied to geoscience and remote sensing," IEEE Transactions on Geoscience and Remote Sensing, vol. 40, pp. 2042-2055, Sept. 2002.

[3] M. Pesaresi and J. A. Benediktsson, "A new approach for the morphological segmentation of high-resolution satellite imagery," IEEE Transactions on Geoscience and Remote Sensing, vol. 39, pp. 309-320, Feb. 2001.

[4] K. Lambers and T. Reitmaier, "Silvretta Historica: Satellite-assisted archaeological survey in an alpine en- vironment," in Proc. of Computer Applications and Quantitative Methods in Archaeology (CAA) Conference, Granada, Spain, April 2010, in Press.

[5] P. Soille, Morphological Image Analysis: Principles and Applications, Springer-Verlag Berlin, 2 edition, 2003.

[6] P. Soille, "Beyond self-duality in morphological image analysis," Image and Vision Computing, vol. 23, pp. 249-257, Feb. 2005.

[7] W. Li, V. Haese-Coat, and J. Ronsin, "Residues of morphological filtering by reconstruction for texture classification," Pattern Recognition, vol. 30, pp. 1081 - 1093, July 1997.

[8] J. Serra and L. Vincent, "An overview of morphological filtering," Circuits, Systems, and Signal Processing, vol. 11, pp. 47-108, 1992.

[9] J. Serra, Image Analysis and Mathematical Morphology, Academic Press, 1982.

[10] N. Otsu, "A Threshold Selection Method from Graylevel Histograms," IEEE Transactions on Systems, Man and Cybernetics, vol. 9, pp. 62-66, Jan. 1979. 